\title{
Towards Road Surface Monitoring: Experiments and Technical Challenges
}

\author{
Jens Eliasson, Wolfgang Birk \\ Dept. of Computer Science and Electrical \\ Engineering \\ Luleå University of Technology \\ S-971 87 Luleå, Sweden \\ jens.eliassoneltu.se
}

\begin{abstract}
This paper discusses results from real-life tests with intelligent road marking units (RMU), placed on the surface of a highway in northern Sweden, to monitor passing vehicles and road properties, as well as making the information available through a wireless sensor network. Wireless sensor networks are starting to gain interest in the area of cooperative safety and efficiency in transport as they can contribute to the reduction of accidents and emissions as well as enhanced driver experiences. Intelligent road markings are another key element for the creation of intelligent transport systems, complementing road side units, devices placed near a road equipped with highperformance computational platforms and long range communication capabilities. This paper shows that RMUs are feasible from the perspectives of low-power consumption, durability, and sensing performance.
\end{abstract}

\section{INTRODUCTION}

Sensor networks are beginning to gain interest in the area of safety and efficiency in transport. This is because traditional measurement systems and sensing devices all have limitations with applications targeting roads, bridges, and tunnels. Wireless sensors on the other hand, with their wireless communication, low power consumption, and low cost, have the potential to improve functions like traffic counting, collision mitigation and avoidance.

Sensor and actuator networks can be seen as a key element in the design of so-called intelligent transport systems (ITS), which are motivated by the societal costs for transport. Fatality in road traffic is the dominating cause for non-natural human death in our societies, [1]. These accidents are the main cause of death in the under 45 age group and cause more deaths than heart disease or cancer in that group. There, an annual cost to society by all traffic accidents is estimated to exceed 160 billion Euro a year, which corresponds to $2 \%$ of EU GNP, [1]. Adding cost for general traffic problems in the EU, i.e. traffic jams, yields a cost to society of $3 \%$ of the EU GNP. Similar figures for the USA can be derived from, [2].

Hence safe and efficient transport solutions, which do not interfere with our need of being mobile, are needed. ITS can be divided into two main streams for road transport: Infrastructure-centric and vehicle-centric. Usually, the infrastructure-centric approach focuses on traffic management and safer roads, while the vehicles centric view focuses on more intelligent vehicles with increased passenger safety, security and protection. Clearly, those two approaches go hand in hand, but are often separated efforts and lead to stand-alone and localized solutions.

Results from a recent study by Ford [3] indicate that stand alone preventive safety systems have limited effect on the overall reduction of accidents. This is in line with a current attempt in the research and development community to integrate both approaches into cooperative ITS enhancing safety and efficiency beyond the limit of stand alone or localized solutions, [4]-[6]. In this context, sensor networks and especially wireless sensor networks, can be the enabling technology to create cost efficient cooperative systems. Moreover, wireless sensor networks offer low-cost deployment opportunities with a short time-to-market.

In this paper, we present a sensor network architecture and results from initial trials with prototype units in a real-life test-bed. The work has been conducted within the framework of the iRoad project [7], which comprises partners from parts supplier for infrastructure, research and authorities, namely Geveko ITS [8], Luleå University of Technology [9] and the Swedish Road Administration [10].

This paper is outlined as follows: Section 2 contains a description of the test setup, as well as the identified requirements. Section 3 presents preliminary results, and the following section 4 presents the conclusions. Finally, ideas for future work are outlined in section 5 .

\section{INITIAL TRIAL SETUP}

The technology pieces to create wireless sensor nodes for a harsh environments like a road surface are actually available. Therefore, the available pieces are put together to RMUs and initially tested. Such initial trials are important to get an understanding of the effects of the environment on the operation, survival and expected life-time of the units.

Additionally, the identification of remaining research, development and deployment challenges is facilitated.

\section{A. Road marking unit}

These units are equipped with LEDs, and are thus capable of providing visual aid to drivers. The markers will turn on their white LEDs when it is dark, and thus assist drivers in seeing how the road extends. Temperature sensors are also used to have the LEDs blink when there is a higher 


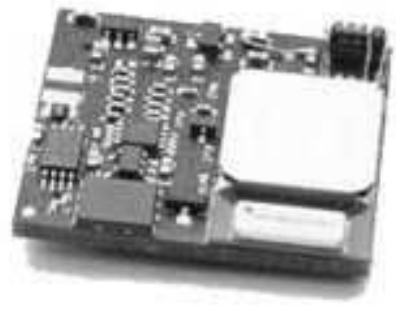

Fig. 1. Eistec Mulle v3 wireless sensor platform

probability of slippery road surface. Since the intelligent road markers are to be deployed on the road surface, their operating conditions are severe. They must be able to be run over by cars, trucks, heavy vehicles, and road maintenance vehicles numerous times. They must be small enough so that they don't interfere with the traffic, i.e. by producing a bumpy road surface, and since a large number of units must be deployed to cover a road, their installation must be done quick, easy, and at a low cost. This prohibits the use of cables as power supply. The road markers used in this project use solar panels and rechargeable batteries.

For easy installation of the trial test system, the Mulle v3 [11] from Eistec [12] was chosen. The Mulle, shown in Fig. 1, is a wirelessly networked sensor node equipped with a Bluetooth 2.0 transceiver. The use of Bluetooth and the TCP/IP protocol suite enables to Mulle to communicate directly with the Internet using standard commercially available access points. The Mulles were encapsulated inside a LEDmark from Geveko ITS. The LEDmark is an electronic road marking powered by solar panels, and its LEDs enables drivers to see how the road extends even in darkness. See Fig. 2 for a photo of an LEDmark with an embedded Mulle device. Deployment of a road marking unit on the road surface is presented in Fig. 3.

\section{B. Access point structure}

For the first trial, an access point consisting of an Asus Eee PC equipped with a radio modem connected to a DLink DBT-900 AP Bluetooth access point was selected. This approach, consisting only of readily available consumer products, allowed the researches to easily provide Internet access to the Mulles. The Eee PC served as gateway to the Internet for the Mulles, as well as providing services such as NTP, databases, and IP address allocation using DHCP. See Fig. 4 for a simplified view of the communication setup during the first trials.

\section{The Mulle networked sensor node}

The Mulle is a Bluetooth-equipped networked sensor node. It was originally developed at EISLAB, Luleå University of Technology (LTU), but is now a commercial product from Eistec AB [12]. The Mulle is based on a Renesas M16C/62P microcontroller with $31 \mathrm{kB}$ of RAM and $384 \mathrm{kB}$

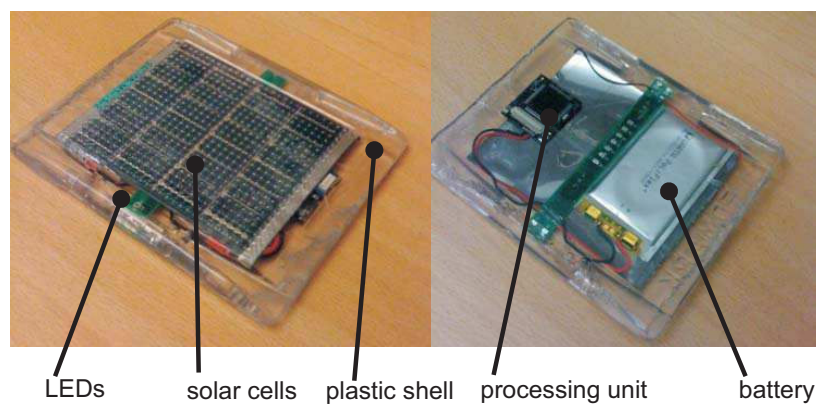

Fig. 2. LEDmark

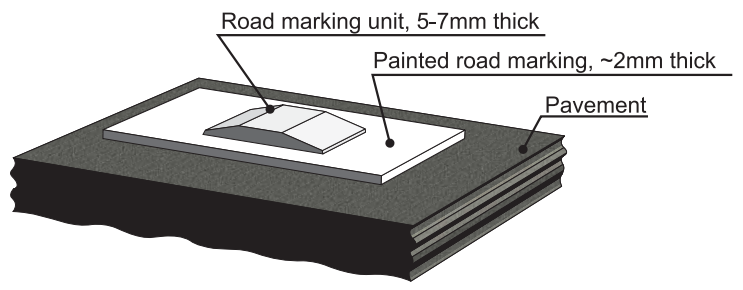

Fig. 3. Sketch over LEDmark

of flash memory, and its communication architecture supports commonly used Bluetooth protocols and profiles.

\section{Communication architecture}

To be able to quickly setup the test system's communication architecture, a choice was made to initially use only consumer products available off the shelf. Internet connection was crucial, since the system is deployed far from any city, and remote monitoring is thus vital. An Asus Eee PC running Ubuntu Linux, equipped with a radio modem, provided all the functionality needed. The Eee PC has no moving parts except for a small fan, and has a relatively low power consumption. The use of the Linux operating system made is easy to setup router functionality, with NAT, DHCP, and NTP services. A D-Link DBT900-AP Bluetooth access point was connected to the Ethernet port on the Eee PC. The Mulles use the Bluetooth access point to communicate with the laptop. The laptop provides the Mulles with IP addresses using DHCP, and Internet access using NAT and the radio modem. The laptop is also used to host a database server, as well as to provide correct date and time to the Mulles using NTP. Some Mulles were configured to access the Internet for NTP and storage of sensor data, while others use the laptop as a server. This approach was chosen so that the stability of the radio modem can be evaluated.

\section{E. Technical challenges}

Deploying a sensor network on the surface of highly trafficked roads requires a number of issues to be managed. The combination of rough weather, heavy traffic, limited energy supply, and hardware and software robustness put high constraints on the system. A classification of the challenges that were detected is outlined below, together with brief descriptions. 


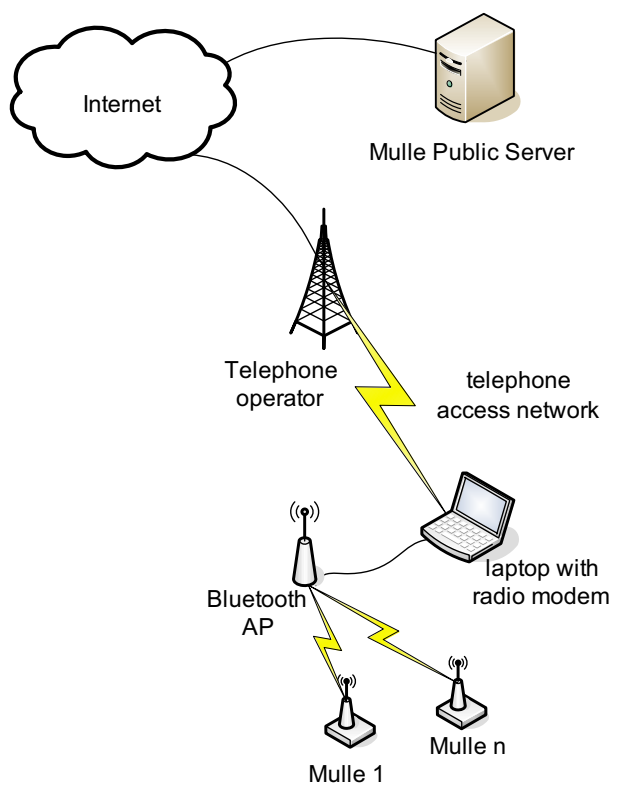

Fig. 4. Communication architecture

1) Harsh environment: Since the nodes in the iRoad project are deployed directly on the road surface of the European highway E4 in northern Sweden, they must be able to cope with traffic in the form of cars, heavy trucks, and road maintenance vehicles such as tractors equipped with snow ploughs. The operation of deploying the nodes on the road requires a great amount of work, making it extremely difficult to exchange faulty sensors.

2) Power supply: Since the nodes are glued directly on the road surface, having someone replacing drained batteries is not a feasible solution. The lifetime is planned to be more than five years, so just using a high-capacity battery is not feasible due to price and size constraints. The only solution is to have the nodes scavenge energy from their environment. For this reason, solar panels were selected since they lack moving parts and can therefore be encapsulated in strong epoxy for protection. However, one problem is that water, dirt, and trash can severely limit the power output from the solar panel, even though the vehicles that passes the RMUs will help keep the panels free.

3) Sensing and Communicating: The type of sensors that can be used is also limited since they must be able be encapsulated in epoxy. This can prohibit the use of some sensor types. One attractive type of sensor for vehicle detection is magnetic sensors, as reported by [13], even though preliminary tests with accelerometers indicate that they can provide more information about passing vehicles than magnetic sensors. When using accelerometers to detect properties of passing vehicles, which have a maximum sensing distance of approximately 10-15 meters. A car passing in $110 \mathrm{~km} / \mathrm{h}$, i.e. $30 \mathrm{~m} / \mathrm{s}$, will only be detectable for $330 \mathrm{~ms}$. This requires the node to sample its sensor at a high rate not to fail detecting a vehicle. And since the network must also be able to detect cars traveling faster than the speed limit, the nodes must sample their sensor at a relatively high rate,

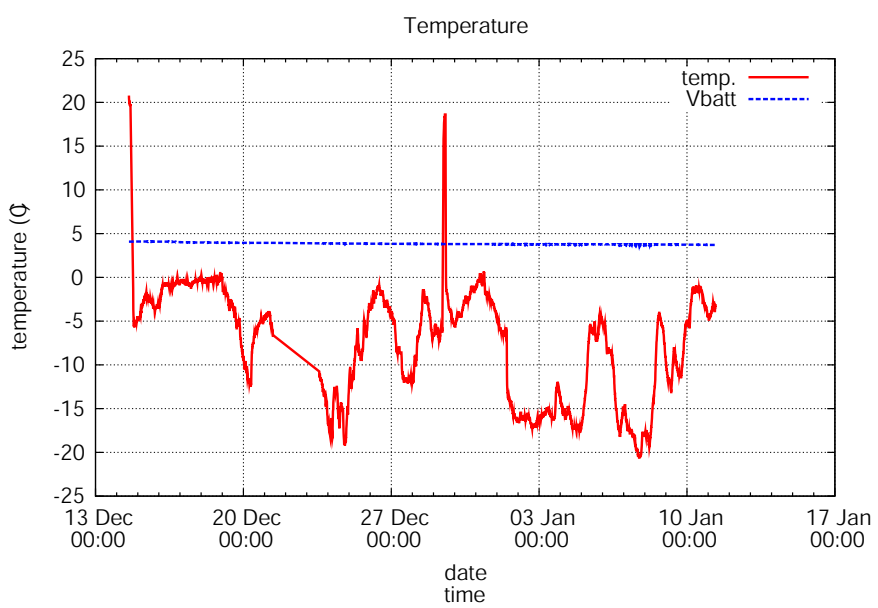

Fig. 5. Outdoor temperature and battery voltage

thus limiting the time they can be in sleep mode to conserve energy. The communication system must also be responsive enough to transmit messages faster than vehicles travel. If nodes are placed every 100 meters, a vehicle can travel that distance in less than two seconds. This means that all nodes must wake up and listen for incoming messages within that time span if messages are supposed to propagate correctly.

\section{Preliminary Results}

A test consisting of four RMUs with integrated Mulles have been carried out during January 2009 to May 2009. The RMUs were placed on a highway, together with an Asus Eee PC as an access point, to investigate how and if low temperatures and bad weather are affecting the Mulles. The battery voltage was monitored to see how the solar cells are performing in low temperatures and under snow. Now, in May, all four Mulles are still operating correctly, and shows no signs of being affected by four months on a winter road, constantly being overrun by cars, trucks, and road maintenance vehicles. The RMU's plastic shells and solar cells are also functioning correctly.

\section{A. Power consumption}

A number of measurements were performed to see the power consumption of the devices. A Mulle in sleep mode consumes only $4 \mu \mathrm{A}$, but when it has its Bluetooth module active, the power consumption is much higher, typically 20-40 mA. The use of renewable energy from solar panels put high demands on the nodes' software [14]. The software should be both energy- and context aware for highest efficiency. Figure 6 shows a successful connection between a Mulle and the Mulle Public Server (MPS) [15] on the Internet. The power consumption includes: powering on Bluetooth module and module initialization, Bluetooth connection to the access point, a DHCP request to obtain an IP address, an NTP request in order to re-synchronize the built-in real-time Clock, and finally a connection against the Mulle Public Server.

In Fig. 7, an example of a failed connection attempt's power consumption and duration is presented. The access 


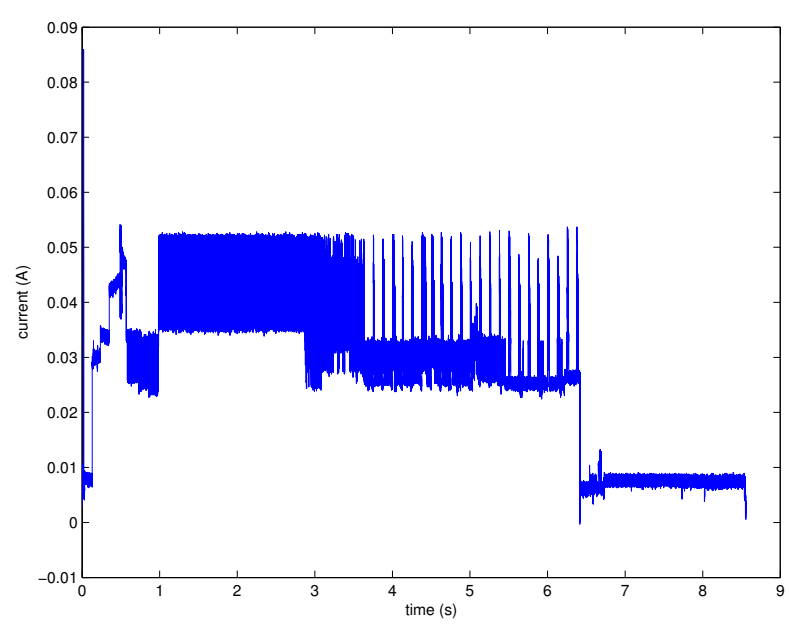

Fig. 6. Power consumption of a successful Internet connection

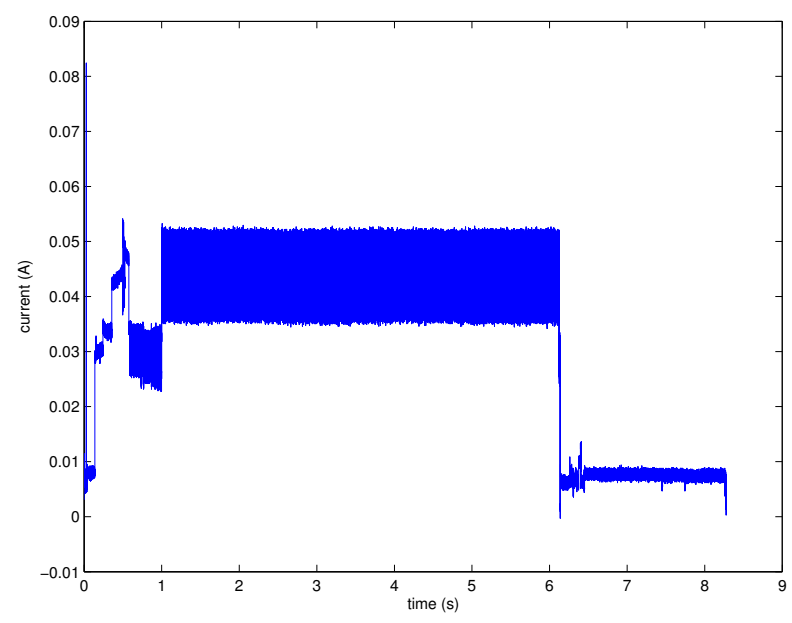

Fig. 7. Power consumption of a failed connection attempt (no AP)

TABLE I

POWER CONSUMPTION

\begin{tabular}{|c||c|}
\hline Communication event type & Energy consumption \\
\hline Successful Internet data transmission & $236 \mathrm{mAs}$ \\
Failed access point connection & $271 \mathrm{mAs}$ \\
Inquiry for new access point & $332 \mathrm{mAs}$ \\
\hline
\end{tabular}

point was powered down in order to simulate a failure of operation and the power consumption was measured of the Mulle as it tries to establish a connection with a non-existing peer.

In Fig. 8, the power consumption and duration of a Bluetooth inquiry is presented. When a Mulle cannot establish a connection for a configurable number of times, at present set to 4, it will perform an Inquiry scan in order to find a new access point. This is done to allow that the access point may break and a replacement is installed. All Mulles will, after performing the procedure described above, start using the new access point automatically.

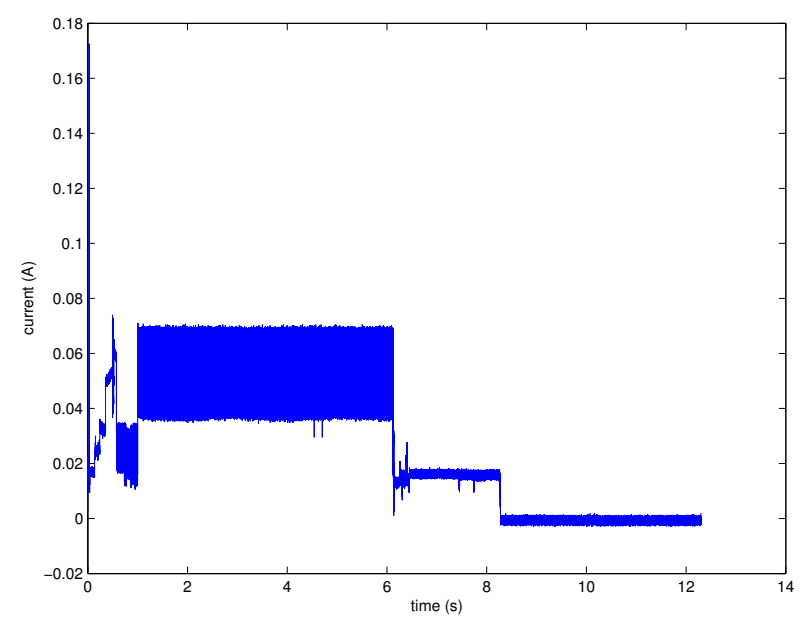

Fig. 8. Power consumption of a Bluetooth Inquiry scan (4 s.)

TABLE II

QUICK FACTS

\begin{tabular}{|c||c|}
\hline Measured parameters & Temperature, battery voltage \\
Sample rate & Every $10 \mathrm{~min}$. \\
Transmission interval & Every hour \\
Battery capacity & $750 \mathrm{mAh}$ \\
Estimated lifetime & More than 1 year \\
\hline
\end{tabular}

\section{B. Robustness}

In order to guarantee correct operation during extensive periods of time in harsh environments, the software was tested for several months before deployment. The communication software was designed in such a way that it can handle virtually all types of errors that can occur. The planned use of IEEE 802.15.4 in the next generation nodes, with less layers and stack complexity, together with the TinyOS [16] operating system will address some of the issues outlined below. Possible communication errors include:

1) Bluetooth access point failure: The access point is unreachable, for any reason. This can be due to a metallic obstacle, i.e. a vehicle, is blocking the transmission, the access point has crashed or because of a power failure. A hardware failure might render it unusable, or that it is so busy server other Mulles that its piconet, which can consist of a maximum of 7 devices, if full.

2) Router failure: In the case where the Asus Eee PC laptop that acts as a router fails, but the Bluetooth access point is up, the Mulles will be able to perform Bluetoothrelated operations, but any TCP/IP-based communication will fail.

3) Internet failure: In this case the Bluetooth connection and IP-address allocation, using DHCP, will be successful. But all attempts to communicate with Internet servers, such as NTP servers and the Mulle Public Server, will fail.

4) Data transmission failure: Even if a connection attempt is successful, a data transfer might still be aborted if a communication failure should occur while sensor data are being transmitted. 


\section{CONCLUSiON}

In this paper, we have presented a sensor node architecture used in real-world trials for the iRoad project. The architecture, based on the Mulle v3 wirelessly networked sensor node, is capable of measuring road surface temperature and transmitting that information directly to the Internet using standardized protocols, i.e. TCP/IP and Bluetooth.

A test setup, consisting of a number of Mulles and a laptop equipped with a radio modem, has been successfully tested outdoors during the cold winter in northern Sweden. Ground temperature, as well as battery status, have been measured and transmitted to the Internet for further processing.

The Mulle, equipped with a solar panel and encapsulated in transparent epoxy in a hard plastic container, brings to life the concept of an intelligent road marking. In the next phase of the iRoad project, a mesh network consisting of large number of intelligent road marking units will create the worlds first intelligent road. The intelligent road will be capable of sensing its surface temperature, and thereby warn approaching vehicles of slippery surfaces. Present work involves adding sensors to the RMUs, thereby enabling them to perform traffic counting using a combination of vibrationand magnetic sensors.

\section{FUTURE WORK}

Work is already in progress to develop an architecture for an upgraded generation sensor nodes as well as a new access point for the next phase of the iRoad project. For that phase, a radio technology supporting mesh networks, i.e. IEEE 802.15.4, will be used together with the TinyOS operating system. Important information, such as power consumption and road influence on the antennas' performance, obtained from the nodes that currently are deployed outdoors in the winter climate of northern Sweden will be used to make the next generation nodes even more robust and thus well-suited for the rough conditions on a winter road carrying heavy traffic.

\section{ACKNOWLEDGMENT}

The authors would like to express their gratitude towards Bruno Hansen at Geveko ITS for his support.

\section{REFERENCES}

[1] European Commission, European Transport policy for 2010: Time to decide. Office For Official Publications Of The European Communities, 2001.

[2] National Highway Traffic Safety Administration, Traffic Safety Facts 2006. US Department of Transportation, 2007, available at: http://www-nrd.nhtsa.dot.gov/Pubs/TSF2006FE.PDF; accessed 200805-09.

[3] B. Gottselig, V. Eis, T. Wey, and R. Sferco, "Entwicklung der verkehrssicherheit, potential bestimmung von modernen sicherheitssystemen im integralen ansatz," in VDA Technischer Kongress 2008, 2. und 3. April, Ludwigsburg, Germany. VDA, Verband der Automobilindustrie, April 2008.

[4] COOPERS, "Co-operative systems for intelligent road safety," http://www.coopers-ip.eu/, 2008, accessed 2009-01-12.

[5] SAFESPOT Integrated Project, "Co-operative systems for road safety: Smart vehicles on smart roads," http://www.safespot-eu.org/, 2008, accessed 2009-01-12.

[6] CVIS, "Cooperative vehicle infrastructure systems," http://www.cvisproject.org/, 2008, accessed 2009-01-12.
[7] iRoad, "Project web site," http://www.iroad.se/, 2009, accessed 200901-12.

[8] GEVEKO, "Company web site," http://www.geveko.se/, 2009, accessed 2009-01-12.

[9] "Luleå University of Technology," 2009, http://www.ltu.se/.

[10] "The Swedish Road Administration," The Swedish Road Administration, 2009, http://www.vv.se/.

[11] J. Eliasson, P. Lindgren, and J. Delsing, "A Bluetooth-based Sensor Node for Low-Power Ad Hoc Networks," Journal of Computers (JCP), May 2008. [Online]. Available: http://www.academypublisher. $\mathrm{com} / \mathrm{jcp} / \mathrm{vol} 103 / \mathrm{no} 05 / \mathrm{jcp} 03050110 . \mathrm{pdf}$

[12] “Eistec AB," Eistec AB, 2008, http://www.eistec.se/.

[13] S.-Y. Cheung, S. C. Ergen, and P. Varaiya, "Traffic surveillance with wireless magnetic sensors," in Proc. 12th ITS World Congress, San Fransisco, USA, November 2005.

[14] J. Eliasson, P. Lindgren, J. Delsing, S. Thompson, and Y.B. Chen, "A Power Management Architecture for Sensor Nodes," in IEEE Wireless Communication and Networking Conference - WCNC 2007, March 2007, pp. 3008-3013.

[15] "EISLAB Mulle Public Server," 2007, http://sm-pc323.sm.ltu.se:8080

[16] “Open-source Operating System," http://www.tinyos.net, March 2008. 